\title{
Quantum Gravity and Recovery of Information in Black Hole Evaporation
}

\author{
Kourosh Nozari $^{\mathrm{a}, \mathrm{b}, *}$ and $\quad$ S. Hamid Mehdipour ${ }^{\mathrm{a}, \mathrm{c}, \dagger}$ \\ ${ }^{a}$ Department of Physics, Faculty of Basic Sciences, University of Mazandaran, \\ P. O. Box 47416-1467, Babolsar, IRAN \\ ${ }^{b}$ Research Institute for Astronomy and Astrophysics of Maragha, \\ P. O. Box 55134-441, Maragha, IRAN \\ ${ }^{c}$ Islamic Azad University, Lahijan Branch, \\ P. O. Box 1616, Lahijan, IRAN
}

\begin{abstract}
The Generalized Uncertainty Principle (GUP), motivated by current alternatives of quantum gravity, produces significant modifications to the Hawking radiation and the final stage of black hole evaporation. We show that incorporation of the GUP into the quantum tunneling process (based on the null-geodesic method) causes correlations between the tunneling probability of different modes in the black hole radiation spectrum. In this manner, the quantum information becomes encrypted in the Hawking radiation, and information can be recovered as non-thermal GUP correlations between tunneling probabilities of different modes.
\end{abstract}

PACS: 04.70.-s, 04.70.Dy

Key Words: Quantum Tunneling, Hawking Radiation, Generalized Uncertainty Principle, Information Loss Paradox

\footnotetext{
*knozari@umz.ac.ir

†h.mehdipour@umz.ac.ir
} 


\section{Introduction}

Quantum gravity proposals commonly predict the existence of a minimum observable length of the order of the Planck length [1-5]. Such a smallest length causes an alteration of the position-momentum uncertainty relation [2] in such a way that $\Delta x \geq \frac{1}{\Delta p}+\alpha L_{p}^{2} \Delta p$, where $\alpha$ is a dimensionless constant of the order of unity that depends on the details of the quantum gravity hypothesis. The above Generalized Uncertainty Principle (GUP) can be obtained from string theory [3], noncommutative quantum theory [4], loop quantum gravity [1], or from black hole gedanken experiments [5]. In the standard limit, $\Delta x \gg L_{p}$, it yields the ordinary uncertainty principle, $\Delta x \Delta p \geq 1$. The GUP becomes susceptible when the momentum and distance scales are close to the Planck scale (see [6,7] and references therein). For a spherical black hole the thermodynamic quantities can be obtained in a heuristic manner using the standard uncertainty principle [8]. Application of the GUP to black hole thermodynamics modifies the results dramatically by incorporation of quantum gravity effects in the final stages of evaporation with a rich phenomenology [9] (see also $[2,10,11]$ ). Implications of the GUP on various problems have been studied extensively some of which can be obtained in Ref. [12].

Parikh and Wilczek [13] constructed a procedure to describe the Hawking radiation emitted from a Schwarzschild black hole as a tunneling process of a massless particle through its quantum horizon. This procedure provides a leading correction to the tunneling probability (emission rate) arising from reduction of the black hole mass due to the energy carried by the emitted quantum. However, due to its lack of correlations between the emission rates of different modes in the black hole radiation spectrum the form of the correction is not adequate by itself to return information. Here we generalize the Parikh-Wilczek tunneling framework by the incorporating quantum gravity effects that are manifested in the existence of minimal observable length. Contrary to our previous work on a non-commutative setup [14], we show that in this case, correlations between the different modes of radiation evolve, which reflect the fact that information emerges continuously during the evaporation process at the quantum gravity level. This feature has the potential to answer some questions regarding the black hole information loss paradox $[15,16]$ and provides a more realistic background for treating the black hole evaporation process in its final stages. 


\section{$2 \quad$ GUP and Parikh-Wilczek Quantum Tunneling}

The first step to discuss the quantum tunneling through the black hole horizon is to find a proper coordinate system for the black hole metric where the constant time slices are flat, and the tunneling path is free of singularities. Painlevé coordinates [17] are suitable choices in this respect. In these coordinates, the Schwarzschild metric is given by

$$
d s^{2}=-\left(1-\frac{2 M}{r}\right) d t^{2}+2 \sqrt{\frac{2 M}{r}} d t d r+d r^{2}+r^{2}\left(d \theta^{2}+\sin ^{2} \theta d \phi^{2}\right),
$$

which is stationary, non-static, and non-singular at the horizon. The radial null geodesics obey

$$
\dot{r} \equiv \frac{d r}{d t}= \pm 1-\sqrt{\frac{2 M}{r}},
$$

where the plus (minus) sign corresponds to outgoing (ingoing) geodesics. Now we incorporate the minimal length scale from quantum gravity via the generalized uncertainty principle which motivates modification of the standard dispersion relation $[2,10]$. AmelinoCamelia et al [2] (see also [18]) studied the black hole evaporation process after an analysis of the GUP-induced modification of the black body radiation spectrum. If GUP is fundamental to quantum gravity, it should appear in de Broglie relation as follows

$$
\lambda \simeq \frac{1}{p}\left(1+\alpha L_{p}^{2} p^{2}\right),
$$

or

$$
\mathcal{E} \simeq E\left(1+\alpha L_{p}^{2} E^{2}\right) .
$$

There are other compelling reasons from noncommutative geometry and loop quantum gravity that support relation (4) (see for instance $[2,10]$ and references therein). With these preliminaries, we consider a massless particle i.e. a shell and take into consideration the response of the background geometry to a radiated quantum of energy $E$ with GUP correction i.e. $\mathcal{E}$. The particle moves on the geodesics of a spacetime with $M-\mathcal{E}$ substituted for $M$. The description of the motion of particles in the $s$-wave as spherical massless shells in a dynamical geometry and the analysis of self-gravitating shells in Hamiltonian gravity have been reported in Refs. [19,20](see also [21]). If one assume that $t$ increases in the direction of the future, then the metric should be modified due to back-reaction effects. We hold the total ADM mass, $M$, of the spacetime fixed but allow the hole mass to fluctuate and replace $M$ by $M-\mathcal{E}$ both in the Painlevé metric and the geodesic equation. Since the characteristic wavelength of the radiation is always arbitrarily small near 
the horizon due to the infinite blue-shift, the wave-number approaches infinity. Therefore the WKB approximation is valid near the horizon. In the WKB approximation, the tunneling probability for the classically inhibited area as function of the imaginary part of the particle action at stationary phase takes the form

$$
\Gamma \sim \exp (-2 \operatorname{Im} I) \approx \exp (-\beta E)
$$

As we will see later, to first order in $E$ the r.h.s of this expression substitutes the Boltzmann factor in the canonical ensemble characterized by the inverse temperature $\beta$. In the $s$-wave picture, particles as spherical massless shells travel on radial null geodesics and transfer across the horizon as outgoing positive energy particles from $r_{\text {in }}$ to $r_{\text {out }}$. The imaginary part of the action is thus given by

$$
\operatorname{Im} I=\operatorname{Im} \int_{r_{\text {in }}}^{r_{\text {out }}} p_{r} d r=\operatorname{Im} \int_{r_{\text {in }}}^{r_{\text {out }}} \int_{0}^{p_{r}} d p_{r}^{\prime} d r
$$

As it is clear from the GUP expression, the commutation relation between the radial coordinate and momentum should be modified as follows [22]

$$
\left[r, p_{r}\right]=i\left(1+\alpha L_{p}^{2} p^{2}\right)
$$

In the classical limit it is replaced by the following Poisson bracket

$$
\left\{r, p_{r}\right\}=1+\alpha L_{p}^{2} p^{2}
$$

Now, using the deformed Hamilton's equation of motion,

$$
\dot{r}=\{r, H\}=\left.\left\{r, p_{r}\right\} \frac{d H}{d p_{r}}\right|_{r}=\left.\left(1+\alpha L_{p}^{2} p^{2}\right) \frac{d H}{d p_{r}}\right|_{r}
$$

where the Hamiltonian is $H=M-\mathcal{E}^{\prime}$, we assume $p^{2} \simeq \mathcal{E}^{\prime 2}$ and eliminate the momentum in favor of energy in the integral (6)

$$
\operatorname{Im} I=\operatorname{Im} \int_{M}^{M-\mathcal{E}} \int_{r_{\text {in }}}^{r_{\text {out }}} \frac{\left(1+\alpha L_{p}^{2} \mathcal{E}^{2}\right)}{\dot{r}} d r d H=\operatorname{Im} \int_{0}^{\mathcal{E}} \int_{r_{\text {in }}}^{r_{\text {out }}} \frac{\left(1+\alpha L_{p}^{2} \mathcal{E}^{2}\right)}{1-\sqrt{\frac{2\left(M-\mathcal{E}^{\prime}\right)}{r}}} d r\left(-d \mathcal{E}^{\prime}\right) .
$$

The $r$ integral can be performed by deforming the contour around the pole at the horizon, where it lies along the line of integration and gives $(-\pi i)$ times the residue

$$
\operatorname{Im} I=\operatorname{Im} \int_{0}^{\mathcal{E}} 4(-\pi i)\left(1+\alpha L_{p}^{2} \mathcal{E}^{\prime 2}\right)\left(M-\mathcal{E}^{\prime}\right)\left(-d \mathcal{E}^{\prime}\right)
$$


This allows us to consider the leading order correction to be just proportional to the square of $\left(\sqrt{\alpha} L_{p}\right)^{\ddagger}$ for simplicity and without loss of generality ${ }^{\S}$. Now the imaginary part of the action takes the form

$$
\operatorname{Im} I=4 \pi M E-2 \pi E^{2}+\pi \alpha L_{p}^{2} E^{3}\left(\frac{16}{3} M-5 E\right)+O\left(\alpha^{2} L_{p}^{4}\right) .
$$

The tunneling rate is therefore

$$
\Gamma \sim \exp \left(-8 \pi M E+4 \pi E^{2}-2 \pi \alpha L_{p}^{2} E^{3}\left(\frac{16}{3} M-5 E\right)+O\left(\alpha^{2} L_{p}^{4}\right)\right)=\exp (\Delta S)
$$

where $\Delta S$ is the difference in black hole entropies before and after emission [13,23]. In string theory it is anticipated that the tunneling rates from excited D-branes in the microcanonical ensemble depends on the final and initial number of microstates available to the system. In a more precise expression, it was shown that the emission rates on the high-energy scale correspond to differences between counting of states in the microcanonical and canonical ensembles [23]. The first and second expressions in the exponential exhibit a similar kind of non-thermal aberration that was discovered in Ref. [13]. So, the emission spectrum cannot be strictly thermal. In our situation, there is an additional term depending on the GUP parameter in first order that cannot be neglected once the black hole mass becomes comparable to the Planck mass.

We now illustrate whether or not, the emission rates for the different modes of radiation during the evaporation are mutually related from a statistical viewpoint. Utilizing (13), the emission rate for a first quantum with energy $E_{1}$, gives

$$
\ln \Gamma_{E_{1}}=-8 \pi M E_{1}+4 \pi E_{1}^{2}-2 \pi \alpha L_{p}^{2} E_{1}^{3}\left(\frac{16}{3} M-5 E_{1}\right) .
$$

Similarly, the emission rate for a second quantum $E_{2}$, takes the form

$$
\ln \Gamma_{E_{2}}=-8 \pi\left(M-E_{1}\right) E_{2}+4 \pi E_{2}^{2}-2 \pi \alpha L_{p}^{2} E_{2}^{3}\left(\frac{16}{3}\left(M-E_{1}\right)-5 E_{2}\right) .
$$

Correspondingly, the emission rate for a single quantum with the same total energy, $E=E_{1}+E_{2}$, yields

$\ln \Gamma_{\left(E_{1}+E_{2}\right)}=-8 \pi M\left(E_{1}+E_{2}\right)+4 \pi\left(E_{1}+E_{2}\right)^{2}-2 \pi \alpha L_{p}^{2}\left(E_{1}+E_{2}\right)^{3}\left(\frac{16}{3} M-5\left(E_{1}+E_{2}\right)\right)$.

\footnotetext{
${ }^{\ddagger}$ This is the minimal length which is governed differently by the parameter $\alpha$ in different alternatives of quantum gravity proposal. For example, in string theory $\alpha$ takes the value which is given by $\alpha \approx \frac{L_{p}^{2}}{\ell_{s}^{2}}$ where $\ell_{s}$ is string length.

${ }^{\S}$ It can be shown easily that forthcoming results of this paper remain credible even without this approximation.
} 
It can be approved that these probabilities are correlated. On the other hand, the non-zero statistical correlation function is

$$
\chi\left(E_{1}+E_{2} ; E_{1}, E_{2}\right)=4 \pi \alpha L_{p}^{2} E_{1} E_{2}\left(-8 M\left(E_{1}+E_{2}\right)+10 E_{1}^{2}+15 E_{1} E_{2}+\frac{22}{3} E_{2}^{2}\right) .
$$

This means that not only the probability of tunneling of two particles of energy $E_{1}$ and $E_{2}$ is not similar to the probability of tunneling of one particle with their compound energies, $E_{1}+E_{2}$, but there are also correlations between them. In fact, whenever one quantum of emission is radiated from the surface of the black hole horizon, the aberrations are created on the Planck scale that influence the second quantum of emission, and these aberrations cannot be neglected, particularly once the black hole mass becomes comparable with the Planck mass. Therefore, as expected in Ref. [24], in this way the form of the amendments as back-reaction effects with incorporation of GUP influences are sufficient by themselves to recover information. Information leaks out from the black hole as the non-thermal GUP correlations within the Hawking radiation.

Currently there are four main proposals about what happens to the information that falls into a black hole. First proposal is that the black hole can evaporate completely as uncorrelated thermal radiation in each mode, and all the information including the original quantum state that formed the black hole, excluding its mass, charge and angular momentum, would disappear from our universe. This means that, in particular, this proposal causes allowing pure states to evolve into mixed states, incompatibility with the basic principles of quantum mechanics. Second proposal is that the black hole can completely disappear, but the information appears in the final burst of radiation when the black hole shrinks to the Planck size. A third possibility is that the black hole never disappears completely, and the information is not lost, but would be stored in a Planck size stable remnant. And fourth idea is that the information comes out in non-thermal correlations within the Hawking radiation, the process being portrayed by a unitary $S$-matrix. In other words, there are non-thermal correlations between different modes of radiation throughout the evaporation process that information emerges ceaselessly encoded through them. In this paper, we have studied the credibility of the fourth conjecture within a perturbative quantum gravity approach. We have shown that equation (17) represents the recovery of the information as real correlations at the Planck scale, where quantum gravity corrections become important.

In summary, the inclusion of the effects of quantum gravity as modification of the de Broglie relation and corresponding commutation relations in the quantum tunneling 
framework of the black hole evaporation, leads to correlation between emitted modes of evaporation. In this setup, information leaks out of the black hole in the form of nonthermal GUP correlations, which might solve the black hole information loss paradox.

\section{Acknowledgment}

We would like to thank Elias Vagenas for useful comments. We would also like to thanks Professor Rudolf Treumann and three anonymous referees for important contribution in this work.

This work has been supported partially by Research Institute for Astronomy and Astrophysics of Maragha, Iran.

\section{References}

[1] L. J. Garay, Int. J. Mod. Phys. A 10 (1995) 145, [arXiv:gr-qc/9403008]

[2] G. Amelino-Camelia et al, Class. Quant. Grav. 23 (2006) 2585, [arXiv:gr-qc/0506110]

[3] D. Amati et al, Phys. Lett. B 216 (1989) 41

[4] M. Maggiore, Phys. Rev. D 49 (1994) 5182, [arXiv:hep-th/9305163]

[5] F. Scardigli, Phys. Lett. B 452 (1999) 39, [arXiv:hep-th/9904025]

[6] K. Nozari, Phys. Lett. B 629 (2005) 41, [arXiv:hep-th/0508078]

[7] S. Hossenfelder, Phys. Rev. D 73 (2006) 105013, [arXiv:hep-th/0603032]

[8] H. Ohanian and R. Ruffini, Gravitation and Spacetime, 2nd ed., p. 481 (W. W. Norton, 1994)

[9] R. J. Adler et al, Gen. Relat. Grav. 33 (2001) 2101, [arXiv:gr-qc/0106080]; A. J. M. Medved and E. C. Vagenas, Phys. Rev. D 70 (2004) 124021, [arXiv:hep-th/0411022]

[10] K. Nozari and A. S. Sefidgar, Phys. Lett. B 635 (2006) 156, [arXiv:gr-qc/0601116]

[11] W. Kim et al, JHEP 01 (2008) 035, [arXiv:0711.0786]; K. Nouicer, Phys. Lett. B 646 (2007) 63, [arXiv:0704.1261]; K. Nozari and S. H. Mehdipour, Mod. Phys. Lett. A 20 (2005) 2937; K. Nozari and S. H. Mehdipour, Int. J. Mod. Phys. A 21 (2006) 1, [arXiv:gr-qc/0511110] 
[12] There is a large literature on this subject; see for example, M. Maggiore, Phys. Lett. B 304 (1993) 65, [arXiv:hep-th/9301067]; K. Nozari and S. H. Mehdipour, Gen. Rel. Grav. 37 (2005) 1995, [arXiv:quant-ph/0507019]; S. H. Mehdipour, K. Nozari and S. D. Sadatian, Fractals 16 (2008) 33; M. Maggiore, Phys. Lett. B 319 (1993) 83, [arXiv:hep-th/9309034]; C. Castro, Found. Phys. Lett. 10 (1997) 273, [arXiv:hepth/9512044]; A. Kempf et al, Phys. Rev. D 52 (1995) 1108, [arXiv:hep-th/9412167]; S. Capozziello et al, Int. J. Theor. Phys. 39 (2000) 15, [arXiv:gr-qc/9910017]; S. Kalyana Rama, Phys. Lett. B 519 (2001) 103, [arXiv:hep-th/0107255]; A. Camacho, Gen. Rel. Grav. 34 (2002) 1839, [arXiv:gr-qc/0206006]; F. Nasseri, Phys. Lett. B 618 (2005) 229, [arXiv:astro-ph/0208222]; M. R. Setare, Phys. Rev. D 70 (2004) 087501, [arXiv:hep-th/0410044]; K. Nozari and S. H. Mehdipour, Chaos, Solitons and Fractals 32 (2007) 1637, [arXiv:hep-th/0601096] and references therein; B. Vakili, Phys. Rev. D 77 (2008) 044023, [arXiv:0801.2438]

[13] M. K. Parikh and F. Wilczek, Phys. Rev. Lett. 85 (2000) 5042, [arXiv:hepth/9907001]

[14] K. Nozari and S. H. Mehdipour, Hawking Radiation as Quantum Tunneling from Noncommutative Schwarzschild Black Hole, Class. Quantum Grav. 25 (2008) 175015, [arXiv:0801.4074]

[15] J. Preskill, Do Black Holes Destroy Information?, Proceeding of International Symposium on Black Holes, Membranes, Wormholes and Superstrings, Houston Advanced Research Center, 16-18 January 1992, Edited by Sunny Kalara and D. V. Nanopoulos, World Scientific, Singapore, 1993, p.22, [arXiv:hep-th/9209058]

[16] D. N. Page, Phys. Rev. Lett. 71 (1993) 3743, [arXiv:hep-th/9306083]

[17] P. Painlevé, Compt. Rend. Acad. Sci. (Paris) 173 (1921) 677

[18] Amelino-Camelia et al, Phys. Rev. D 70 (2004) 107501, [arXiv:gr-qc/0405084]

[19] P. Kraus and F. Wilczek, Nucl. Phys. B 433 (1995) 403, [arXiv:gr-qc/9408003]

[20] E. C. Vagenas, Phys. Lett. B 503 (2001) 399, [arXiv:hep-th/0012134]; E. C. Vagenas, Mod. Phys. Lett. A 17 (2002) 609, [arXiv:hep-th/0108147]; E. C. Vagenas, Phys. Lett. B 533 (2002) 302, [arXiv:hep-th/0109108]; A. J. M. Medved, Class. Quant. Grav. 19 (2002) 589, [arXiv:hep-th/0110289]; A. J. M. Medved, Phys. Rev. D 66 
(2002) 124009, [arXiv:hep-th/0207247]; E. C. Vagenas, Phys. Lett. B 559 (2003) 65, [arXiv:hep-th/0209185]; M. K. Parikh, Int. J. Mod. Phys. D 13 (2004) 2351, [arXiv:hep-th/0405160]

[21] S. Shankaranarayanan et al, Mod. Phys. Letts. A 16 (2001) 571, [arXiv:grqc/0007022]; E. C. Vagenas, Nuovo Cim. B 117 (2002) 899, [arXiv:hep-th/0111047]

[22] See for instance, S. Benczik et al, Phys. Rev. D 66 (2002) 026003, [arXiv:hepth/0204049]

[23] E. Keski-Vakkuri and P. Kraus, Nucl. Phys. B 491 (1997) 249, [arXiv:hepth/9610045]

[24] M. Arzano et al, JHEP 0509 (2005) 037, [arXiv:hep-th/0505266]; A. J. M. Medved and E. C. Vagenas, Mod. Phys. Lett. A 20 (2005) 1723, [arXiv:gr-qc/0505015]; A. J. M. Medved and E. C. Vagenas, Mod. Phys. Lett. A 20 (2005) 2449, [arXiv:grqc/0504113] 